九州大学学術情報リポジトリ

Kyushu University Institutional Repository

\title{
Diffusion coefficients of cetyl alcohol in supercritical carbon dioxide
}

Higashi, Hidenori

Division of Material Engineering, Graduate School of Natural Science and Technology, Kanazawa University

Iwai, Yoshio

Department of Chemical Engineering, Faculty of Engineering, Kyushu University

Oda, Tsuyoshi

Department of Chemical Engineering, Faculty of Engineering, Kyushu University

Murai, Kengo

Department of Chemical Engineering, Faculty of Engineering, Kyushu University

他

http://hdl. handle. net/2324/12523

出版情報: Journal of Chemical Engineering of Japan. 38 (11)，pp.865-869，2005-11-20. 化学工学 会

バージョン：

権利関係: (C) 2005 The Society of Chemical Engineers, Japan. 


\title{
Diffusion Coefficients of Cetyl Alcohol in Supercritical Carbon Dioxide
}

\author{
Hidenori Higashi ${ }^{1}$, Yoshio IWAI $^{2}$, Tsuyoshi OdA ${ }^{2}$, \\ Kengo MURAI ${ }^{2}$ and Yasuhiko ARAI ${ }^{2}$ \\ ${ }^{1}$ Division of Material Engineering, Graduate School of Natural \\ Science and Technology, Kanazawa University, Kakuma-machi, \\ Kanazawa-shi, Ishikawa 920-1192, Japan \\ ${ }^{2}$ Department of Chemical Engineering, Faculty of Engineering, \\ Kyushu University, 10-1, Hakozaki 6, Higashi-ku, Fukuoka-shi, \\ Fukuoka 812-8581, Japan
}

Keywords: Experiment, Molecular Simulation, Diffusion Coefficient, Supercritical Carbon Dioxide

\begin{abstract}
The diffusion coefficients of cetyl alcohol in supercritical carbon dioxide were measured by the pseudo steady state solid dissolution method at $308.2 \mathrm{~K}$. The Schmidt number correlation was applied to correlate with the experimental diffusion coefficients. Further, molecular dynamics simulation was performed to calculate the diffusion coefficients by an all atom model of solute. The correlated results by the Schmidt number correlation show good agreement with the experimental results. The calculated results by molecular dynamics simulation give fair estimation to the experimental data without adjustable parameters.
\end{abstract}

\section{Introduction}

The supercritical fluid extraction has been given much attention recently as one of the new separation technologies in the chemical industry. It is very useful if natural products can be separated and purified with high selectivity. One of the important physical properties necessary for designing a supercritical extractor, a separator and a reactor is a diffusion coefficient in the supercritical fluids. The diffusion data of natural products in supercritical gas are essentially important in the process design of the supercritical fluid extraction. However, the measurement of diffusion coefficients under the supercritical condition is difficult so that the diffusion coefficient data in the supercritical region are quite limited. The Taylor dispersion method (Taylor, 1953; Aris, 1956) has been the most useful technique to measure the diffusion coefficients in supercritical fluids (Swaid and Schneider, 1979; Liong et al., 1991; Suárez et al., 1998). The modified Taylor dispersion method (Funazukuri et al., 2002) and the chromatographic impulse response method (Funazukuri et al., 2000; Kong et al., 2004) were developed to measure diffusion coefficients. These methods were reviewed by Funazukuri et al. (2004). In previous works (Higashi et al., 1998a, 1999), the authors applied a pseudo steady state solid dissolution method (Knaff and Schlünder,

Received on March 14, 2005. Correspondence concerning this article should be addressed to H. Higashi (E-mail address: higashi@t.kanagawa-u.ac.jp).
1987) to measure the diffusion coefficients of aromatic compounds in supercritical carbon dioxide. In this work, the diffusion coefficients of cetyl alcohol in supercritical carbon dioxide, which is one of the natural products, were measured by the pseudo steady state solid dissolution method. The solubility data are necessary to determine the diffusion coefficients by this method. The available solubility data of cetyl alcohol were already reported by Iwai et al. (1991). Thus, cetyl alcohol was selected as a solute in the present study. The diffusion coefficients were correlated by the Schmidt number correlation (Funazukuri et al., 2004).

On the other hand, the molecular simulation may be also feasible and helpful to obtain the thermodynamic properties for mixtures under high pressure. The authors applied a molecular dynamics simulation to calculate the diffusion coefficients of aromatic compounds in supercritical carbon dioxide (Iwai et al., 1997; Higashi et al., 1998b). In this work, the diffusion coefficients of cetyl alcohol in supercritical carbon dioxide were simulated by the molecular dynamics simulation.

\section{Experimental}

\subsection{Materials}

Cetyl alcohol of reagent grade (approximately 99 mol\% supplied by Sigma-Aldrich Co.) was used as received without further purification. High-purity carbon dioxide, with the minimum purity of $99.999 \%$, was supplied by Sumitomo Seika Chemicals Co., Ltd. 


\subsection{Apparatus and procedure}

The pseudo steady state solid dissolution method (Knaff et al., 1987) was adopted to measure the diffusion coefficients of cetyl alcohol in supercritical carbon dioxide. The experimental apparatus and details of the procedure were reported previously (Higashi et al., 1998a, 1999). The diffusion coefficients can be obtained by the following equation.

$$
D_{21}=\frac{\rho_{2}\left(h_{\mathrm{e}}^{2}-h_{\mathrm{b}}^{2}\right)}{2 m_{2} C y_{2}^{\text {sat }} \Delta t}
$$

where $\rho_{2}$ and $m_{2}$ are the packed mass density and the molar mass of solid solute, respectively. $h_{\mathrm{b}}$ and $h_{\mathrm{e}}$ are the distances from the entrance of capillary to the surface of the solid solute at the beginning and the end of the experiment and $\Delta t$ is the elapsed time. $C$ and $y_{2}$ sat are the molar density and saturated solubility for the system. $h_{\mathrm{b}}, h_{\mathrm{e}}$ and $\rho_{2}$ were determined by using a micrometer with the scale of $10^{-3} \mathrm{~mm}$ and an electric balance with the scale of $10^{-5} \mathrm{mg}$. The molar densities of the mixture were replaced by the molar densities of pure carbon dioxide calculated by the equation of Angus et al. (1976). The saturated solubilities for cetyl alcohol in supercritical carbon dioxide were cited from the data of Iwai et al. (1991).

\section{Correlation}

The Schmidt number correlation proposed by Funazukuri et al. (2004) was used to calculate the diffusion coefficients. The diffusion coefficient at the infinite dilution condition is represented by the following equation by definition of the Schmidt number.

$$
D_{21}=\frac{1}{S c}\left(\frac{\mu_{1}}{\rho_{1}}\right)
$$

The density and viscosity of the solvent are given by those of pure carbon dioxide calculated by the equation of Angus et al. (1976) and Chung et al. (1988), respectively.

The Schmidt number correlation is given by

$$
S c=S c^{+} \cdot S c^{*}
$$

$\mathrm{Sc}^{+}$is associated with the molar volume of the solvent by the following equation.

$$
\ln \left(S c^{+}-1\right)=\sum_{i=0}^{5} a_{i}\left(\frac{v_{0}}{v}\right)^{i}
$$

where $v$ is the molar volume of the solvent, $v_{0}$ is the closed-packed hard sphere volume of the solvent, and
Table 1 Parameters for Schmidt number correlation

\begin{tabular}{lcl}
\hline Component & $v_{0}\left[\mathrm{~m}^{3} \mathrm{~mol}^{-1}\right]$ & $d_{\mathrm{vdw}}[\mathrm{nm}]$ \\
\hline Carbon dioxide & $19.7 \times 10^{-6 *}$ & $0.397 * *$ \\
Cetyl alcohol & & $0.822 * *$ \\
\hline
\end{tabular}

*calculated by the method of Funazukuri et al. (1992) at $308.2 \mathrm{~K}$

**calculated by the method of Bondi (1964)

$a_{i}$ is the coefficient $\left(a_{0}=0.284093, a_{1}=2.55177\right.$, $\left.a_{2}=-2.26978, a_{3}=0.756249, a_{4}=-0.08903\right)$ determined by Funazukuri et al. (2004).

The closed-packed hard sphere volume of the solvent was calculated from the polynomial function of temperature proposed by Funazukuri et al. (1992).

$$
v_{0}=\frac{1}{1.384} \sum_{i=0}^{4} b_{i} T^{i}
$$

where $b_{0}=4.452 \times 10^{-5}, b_{1}=-1.152 \times 10^{-7}, b_{2}=2.749$ $\times 10^{-10}, b_{3}=-3.073 \times 10^{-13}, b_{4}=1.290 \times 10^{-16}$.

In Eq. (3), $S c^{*}=5 / 6$ for self-diffusion. For binary diffusion, the following equation can be used.

$$
S c^{*}=\frac{5}{6}\left(\frac{d_{1}+d_{2}}{2 d_{1}}\right)^{2}\left(\frac{2 M_{2}}{M_{1}+M_{2}}\right)^{1 / 2}
$$

where $d$ and $M$ are the effective hard-sphere diameter and the molecular weight, respectively.

The ratio of effective hard-sphere diameters of solvent (1) to solute (2) was assumed by

$$
\frac{d_{1}}{d_{2}}=\frac{d_{\mathrm{vdw}, 1}}{d_{\mathrm{vdw}, 2}}
$$

where $d_{\mathrm{vdw}}$ is the van der Waals diameter estimated by the method of Bondi (1964). The parameters of the Schmidt number correlation are listed in Table 1.

\section{Simulation}

\subsection{Models and potential functions}

Carbon dioxide was treated as a Lennard-Jones molecule (single site model) and cetyl alcohol was treated as a flexible model of all atoms (all atom model). The Lennard-Jones (12-6) potential function was used for all sites in the simulation,

$$
\phi\left(r_{i j}\right)=4 \varepsilon_{i j}\left\{\left(\frac{\sigma_{i j}}{r_{i j}}\right)^{12}-\left(\frac{\sigma_{i j}}{r_{i j}}\right)^{6}\right\}
$$


Table 2 Potential parameters

\begin{tabular}{lcr}
\hline Site & $\sigma[\mathrm{nm}]$ & $\varepsilon / k[\mathrm{~K}]$ \\
\hline Carbon dioxide* & 0.372 & 236.1 \\
Cetyl alcohol** & & \\
C & 0.343 & 52.9 \\
H & 0.257 & 22.2 \\
O & 0.312 & 30.2 \\
\hline *Iwai et al. (1997) & & \\
**universal force field (Rappe et al., & 1992)
\end{tabular}

where $\phi$ is the intermolecular potential, $\varepsilon$ is the energy parameter, $\sigma$ is the size parameter, $r$ is the distance, and $i$ and $j$ denote the particles $i$ and $j$, respectively.

The potential parameters for the single site model of carbon dioxide were referred from the model proposed by Iwai et al. (1997). These parameters were determined by the $P V T$ behavior of pure carbon dioxide. The parameters for the all atom model of the solute were adopted from the universal force field (Rappe et al., 1992) in the Cerius ${ }^{2}$ software (Accelrys Software Inc.). The charge of the solute molecules was unconsidered and the conformation of the solute molecule was optimized by minimizing the energy. All the parameters used in the present study are listed in Table 2 . The arithmetic and geometric combining rules were used respectively to calculate the size and energy parameters between unlike molecules.

\subsection{Simulation conditions and analysis}

An $N V T$ ensemble MD simulation was applied to calculate the diffusion coefficients of carbon dioxide and cetyl alcohol in supercritical carbon dioxide. The time step of the calculations was set to $1 \mathrm{fs}$. The system consisted of 300 carbon dioxide molecules and one cetyl alcohol molecule. The simulation steps were $1.0 \times 10^{4}$ for equilibrium and were $1.0 \times 10^{6}$ for production. The cut-off length was set to the half-cell. The calculated diffusion coefficients are considered as the infinite dilution diffusion coefficients of the cetyl alcohol in supercritical carbon dioxide. The diffusion coefficients for carbon dioxide, $D_{11}$, and cetyl alcohol, $D_{21}$, were calculated by the mean square displacement (MSD) of the center of mass for the molecules,

$$
\begin{aligned}
& D_{11}=\lim _{t \rightarrow \infty} \frac{1}{6 t}\left\langle\left[\mathbf{r}_{1}(t)-\mathbf{r}_{1}(0)\right]^{2}\right\rangle \\
& D_{21}=\lim _{t \rightarrow \infty} \frac{1}{6 t}\left\langle\left[\mathbf{r}_{2}(t)-\mathbf{r}_{2}(0)\right]^{2}\right\rangle
\end{aligned}
$$

where $\mathbf{r}$ is the position of a particle and $t$ is time. The diffusion coefficients were determined by the slope of MSD from 2 to $10 \mathrm{ps}$.
Table 3 Experimental results for diffusion coefficients of cetyl alcohol in supercritical carbon dioxide at $308.2 \mathrm{~K}$

\begin{tabular}{llll}
\hline$P[\mathrm{MPa}]$ & $C \times 10^{6}\left[\mathrm{~mol} \mathrm{~m}^{-3}\right]^{*}$ & $y_{2}^{\text {sat }}[-]^{* *}$ & $D_{21} \times 10^{8}\left[\mathrm{~m}^{2} \mathrm{~s}^{-1}\right]$ \\
\hline 10.6 & 0.01687 & 0.00101 & 0.807 \\
12.8 & 0.01780 & 0.00164 & 0.701 \\
15.8 & 0.01877 & 0.00209 & 0.637 \\
18.7 & 0.01944 & 0.00270 & 0.618 \\
\hline
\end{tabular}

*calculated by equation of state of Angus et al. (1976)

**Iwai et al. (1991)

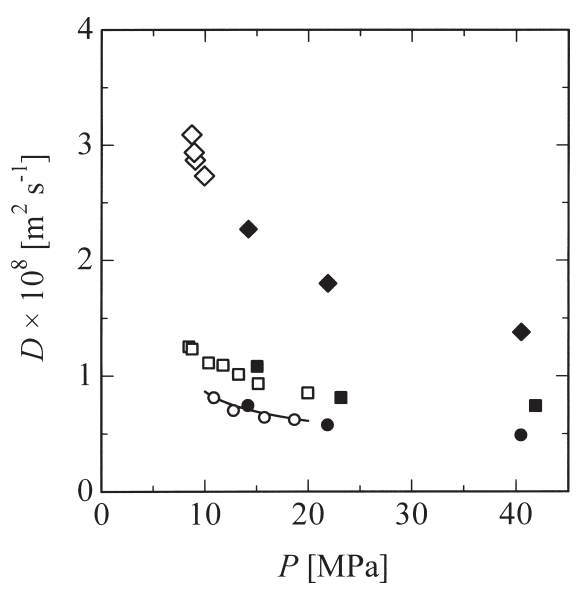

Fig. 1 Diffusion coefficients of carbon dioxide and cetyl alcohol in supercritical carbon dioxide as a function of pressure at $308.2 \mathrm{~K}$. Experimental: $(\diamond)$ carbon dioxide, O'hern and Martin (1955); ( $\square$ ) naphthalene, Higashi et al. (1999); (○) cetyl alcohol, this work; Schmidt number correlation: (-) cetyl alcohol; MD calculations: $(\diamond)$ carbon dioxide, (ם) naphthalene, Higashi et al. (2005); (O) cetyl alcohol, this work

\section{Results and Discussion}

The experimental results did not depend on the flow rate of carbon dioxide. The diffusion coefficients were determined from an arithmetic average of several measurements and the maximum deviation from the average value was within $10 \%$. The reproducibilities were within $5 \%$. The experimental results for diffusion coefficients of cetyl alcohol in supercritical carbon dioxide at $308.2 \mathrm{~K}$ were listed in Table 3 and illustrated in Figure 1. The diffusion coefficients smoothly decrease as pressure increases, because the density of carbon dioxide increases as pressure increases. Fulton et al. (1991) reported that alcohol does not aggregate in supercritical carbon dioxide at dilute conditions. In the present work, the experimental results for diffusion coefficients of cetyl alcohol are about one third of those of carbon dioxide and are about 30\% smaller than the results of naphthalene measured by 
the same apparatus (Higashi et al., 1999), because the size of the cetyl alcohol molecule is larger than that of the naphthalene molecule. The diameter of cetyl alcohol used for the Schmidt number correlation is about 1.3 times larger than that of naphthalene and the molecular weight of cetyl alcohol is about 1.9 times larger than that of naphthalene.

The correlated results by the Schmidt number correlation show good agreement with the experimental results as shown in Figure 1. The availability of the Schmidt number correlation was proved for the chain molecule.

The simulated results of diffusion coefficients of carbon dioxide and cetyl alcohol are shown in Figure 1. The simulated results for carbon dioxide smoothly admit to the experimental results. The simulated results for cetyl alcohol by the all atom model are fair estimations to the experimental data without adjustable parameters. The simulated results for naphthalene by the all atom model (Higashi et al., 2005) were also fair estimation to the experimental results.

The molecular shapes of naphthalene and cetyl alcohol are very different from each other. Naphthalene is a flat molecule and cetyl alcohol is a chain molecule. However, the diffusion coefficients of both molecules are well represented by the Schmidt number correlation and by the molecular simulation with the all atom model. The molecular shapes would be reflected by these models.

\section{Conclusions}

The diffusion coefficients of cetyl alcohol in supercritical carbon dioxide were measured by a pseudo steady state solid dissolution method. The experimental results were correlated by the Schmidt number correlation. Further, the molecular dynamics simulation was performed to calculate the diffusion coefficients. The Schmidt number correlation is available to correlate the diffusion coefficients of chain molecules, such as cetyl alcohol. The diffusion coefficients of cetyl alcohol in supercritical carbon dioxide by molecular dynamics simulations represent reasonable results against the experimental data without adjustable parameters for a binary system.

\begin{tabular}{|c|c|c|}
\hline \multicolumn{3}{|c|}{ Nomenclature } \\
\hline$C$ & $=$ & molar density \\
\hline$D$ & $=$ & diffusion coefficient \\
\hline$d$ & $=$ & diameter of a molecule \\
\hline$h$ & $=$ & $\begin{array}{l}\text { distances from the entrance of a capillary to the } \\
\text { surface of solid solute } \\
\text { [m] }\end{array}$ \\
\hline$M$ & $=$ & molecular weight $\quad[-]$ \\
\hline$m$ & $=$ & molar mass \\
\hline$P$ & $=$ & pressure \\
\hline$r$ & $=$ & distance between molecules \\
\hline $\mathbf{r}$ & $=$ & position of a particle \\
\hline$t$ & $=$ & time \\
\hline$y$ & $=$ & solubility (mole fraction) \\
\hline
\end{tabular}

$\begin{array}{lllr}\Delta t & =\text { elapsed time } & {[\mathrm{s}]} \\ \varepsilon & =\text { energy parameter } & {[\mathrm{J}]} \\ \rho & = & \text { packed mass density } & {\left[\mathrm{kg} \mathrm{m}^{-3}\right]} \\ \sigma & =\text { size parameter } & {[\mathrm{m}]} \\ \phi & =\text { potential } & {[\mathrm{J}]}\end{array}$

$<$ Subscript $>$

$\begin{array}{ll}\mathrm{b} & =\text { beginning of the experiment } \\ \mathrm{e} & =\text { end of the experiment } \\ i, j & =\text { molecules } i \text { and } j \\ \mathrm{sat} & =\text { saturated } \\ \mathrm{vdw} & =\text { van der Waals } \\ 1 & =\text { carbon dioxide } \\ 2 & =\text { cetyl alcohol }\end{array}$

\section{Literature Cited}

Angus, S., B. Armstrong and K. M. de Reuck eds.; Carbon Dioxide, IUPAC Chemical Data Series vol. 3, Project Center, International Union of Pure and Applied Chemistry, Division of Physical Chemistry, Commission on Thermodynamics and Thermochemistry, Thermodynamic Tables Project, Pergamon Press, New York, U.S.A. (1976)

Aris, R.; "On the Dispersion of Solute in Fluid Flowing through a Tube," Proc. Roy. Soc., 235, 67-77 (1956)

Bondi, A.; "Van der Waals Volumes and Radii," J. Phys. Chem., 68, 441-451 (1964)

Chung, T.-H., M. Ajlan, L. L. Lee and K. E. Starling; "Generalized Multiparameter Correlation for Nonpolar and Polar Fluid Transport Properties," Ind. Eng. Chem. Res., 27, 671-679 (1988)

Fulton, J. L., G. G. Yee and R. D. Smith; "Hydrogen Bonding of Methyl Alcohol-d in Supercritical Carbon Dioxide and Supercritical Ethane Solutions," J. Am. Chem. Soc., 113, 83278334 (1991)

Funazukuri, T., Y. Ishiwata and N. Wakao; "Predictive Correlation for Binary Diffusion Coefficients in Dense Carbon Dioxide," AIChE J., 38, 1761-1768 (1992)

Funazukuri, T., C. Y. Kong, N. Morooka and S. Kagei; "Measurements of Binary Diffusion Coefficients and Partition Ratios for Acetone, Phenol, $\alpha$-Tocopherol, and $\beta$-Carotene in Supercritical Carbon Dioxide with a Poly(Ethylene Glycol)-Coated Capillary Column," Ind. Eng. Chem. Res., 39, 4462-4469 (2000)

Funazukuri, T., C. Y. Kong and S. Kagei; "Measurements of Binary Diffusion Coefficients for Some Low Volatile Compounds in Supercritical Carbon Dioxide by Input-Output Response Technique with Two Diffusion Columns Connected in Series," Fluid Phase Equilib., 194-197, 1169-1178 (2002)

Funazukuri, T., C. Y. Kong and S. Kagei; "Impulse Response Techniques to Measure Binary Diffusion Coefficients under Supercritical Conditions," J. Chromatogr., A, 1037, 411-429 (2004)

Higashi, H., Y. Iwai, Y. Takahashi, H. Uchida and Y. Arai; "Diffusion Coefficients of Naphthalene and Dimethylnaphthalene in Supercritical Carbon Dioxide," Fluid Phase Equilib., 144, 269278 (1998a)

Higashi, H., Y. Iwai, H. Uchida and Y. Arai; "Diffusion Coefficients of Aromatic Compounds in Supercritical Carbon Dioxide Using Molecular Dynamics Simulation," J. Supercrit. Fluid., 13, 93-97 (1998b)

Higashi, H., Y. Iwai, Y. Nakamura, S. Yamamoto and Y. Arai; “Correlation of Diffusion Coefficients for Naphthalene and Dimethylnaphthalene Isomers in Supercritical Carbon Dioxide," Fluid Phase Equilib., 166, 101-110 (1999)

Higashi, H., Y. Iwai and Y. Arai; "Comparison of Molecular Models Used in Molecular Dynamics Simulation for Tracer Diffusion Coefficients of Naphthalene and Dimethylnaphtalene Isomers in Supercritical Carbon Dioxide," Fluid Phase Equilib., 234, 51-55 (2005) 
Iwai, Y., T. Fukuda, Y. Koga and Y. Arai; "Solubilities of Myristic Acid, Palmitic Acid, and Cetyl Alcohol in Supercritical Carbon Dioxide at $35^{\circ}$ C," J. Chem. Eng. Data, 36, 430-432 (1991)

Iwai, Y., H. Higashi, H. Uchida and Y. Arai; "Molecular Dynamics Simulation of Diffusion Coefficients of Naphthalene and 2-Naphthol in Supercritical Carbon Dioxide," Fluid Phase Equilib., 127, 251-261 (1997)

Knaff, G. and E. U. Schlünder; "Diffusion Coefficients of Naphthalene and Caffeine in Supercritical Carbon Dioxide," Chem. Eng. Process, 21, 101-105 (1987)

Kong, C. Y., T. Funazukuri and S. Kagei; "Chromatographic Impulse Response Technique with Curve Fitting to Measure Binary Diffusion Coefficients and Retention Factors Using Polymer-Coated Capillary Columns," J. Chromatogr., A, 1035, 177193 (2004)

Liong, K. K., P. A. Wells and N. R. Foster; "Diffusion Coefficients of Long-Chain Esters in Supercritical Carbon Dioxide," Ind.
Eng. Chem. Res., 30, 1329-1335 (1991)

O'hern, H. A., Jr. and J. J. Martin; "Diffusion in Carbon Dioxide at Elevated Pressures," Ind. Eng. Chem., 47, 2081-2087 (1955)

Rappe, A. K., C. J. Casewit, K. S. Colwell, W. A. Goddard III and W. M. Skiff; "UFF, a Full Periodic-Table Force-Field for Molecular Mechanics and Molecular-Dynamics Simulations," J. Am. Chem. Soc., 114, 10024-10035 (1992)

Suárez, J. J., I. Medina and J. L. Bueno; "Diffusion Coefficients in Supercritical Fluids: Available Data and Graphical Correlations," Fluid Phase Equilib., 153, 167-212 (1998)

Swaid, I. and G. M. Schneider; "Determination of Binary Diffusion Coefficients of Benzene and Some Alkylbenzene in Supercritical $\mathrm{CO}_{2}$ between 308 and $328 \mathrm{~K}$ in the Pressure Range 80 to 160 bar with Supercritical Fluid Chromatography (SFC)," Ber. Bunsenges. Phys. Chem., 83, 969-974 (1979)

Taylor, G.; "Dispersion of Soluble Matter in Solvent Flowing Slowly through a Tube," Proc. Roy. Soc., 219, 186-203 (1953) 